
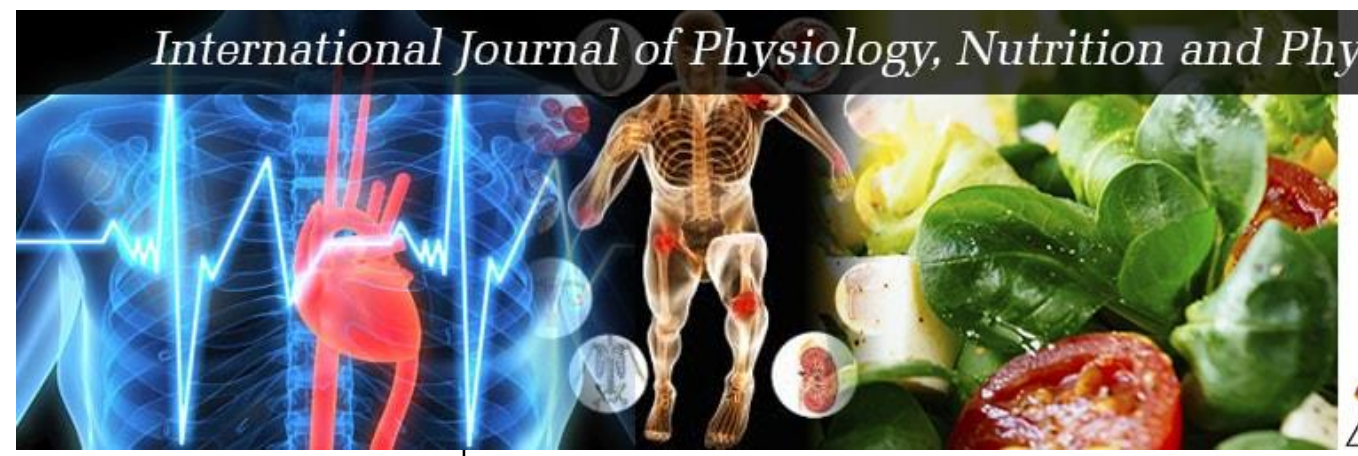

\title{
ysical Education
}

ISSN: $2456-0057$

IJPNPE 2021; 6(2): 213-214

(C) 2021 IJPNPE

www.journalofsports.com

Received: 14-05-2021

Accepted: 18-06-2021

Mamidi Sharath

Physical Director, Dr. B. R. Ambedkar Jr. College Bagh

Lingampally, Hyderabad,

Telangana, India
Corresponding Author: Mamidi Sharath

Physical Director, Dr. B. R. Ambedkar Jr. College Bagh Lingampally, Hyderabad,

Telangana, India

\section{A study on aggressiveness between male and female sportsperson of all Indiainter university players}

\section{Mamidi Sharath}

DOI: https://doi.org/10.22271/journalofsport.2021.v6.i2d.2371

\section{Abstract}

The paper is an empirical attempt to explore the influence of sex variables on aggressive behaviour of male and female sportsperson of All India Inter-University players. Sample of 200 sportsperson in the age group of 19 to 25 years studied by using semi-structured interview schedule. It is explored that impact of sex factor on the sports person has something to do with the aggressive behaviours of sportsperson. But results reveal that there is no significant difference in their aggressive behaviour of male and female sportsperson.

Keywords: Sex variables, competitive sport, sex differences, high and low aggression, socio-economic status, aggressive behaviour

\section{Introduction}

There has always been interest in aggressive behaviour and competitive sport. Several writers have maintained that sport provides a necessary "safety value" or cathartic release for the aggressive drives of the human (Scott, 1970; Lorenz, 1966). Opponents of this position have posited that successful aggressive behaviour facilities further aggressive behaviour. Controlled research findings support this view and many writers have stated that sport should not be compromised as an excuse for punitive and criminal - like behaviour (McMurty, 1974; Underwood, 1978; Vaz, 1976). The arguments for and against aggression and violence in sport are often emotional debates. A major obstacle encountered in this discussion is that the discussants are frequently not talking about the same concept even though they are using the same term! When one begins to examine the various writings on aggressive behaviour, it is clear that the term aggression has the misfortune of being defined in a multitude of ways. The use and misuse of the term is often confusing to players, coaches, and researchers. What does one mean by aggression or aggressiveness? Since these terms have taken popular connotations; they are often used to describe different classes of behaviour rather than a unitary concept. Unfortunately, research on aggressive behaviour in sport has also been plagued by inconsistencies and vague definitions of "aggression" both as an independent and dependent variable. It appears that a clarification of the term aggression will benefit all who are interested in furthering the understanding of aggression and athletic performance. A study completed by Sliva (1979a) found that subjects exhibiting hostile aggression in a sport setting experienced significantly lower levels of guilt than did subjects exhibiting similar behaviour in a non-sport setting. Subjects in the sport setting were also not able to discriminate between appropriate and inappropriate behaviour whereas subjects in the non-sport setting were able to discriminate. This finding is consistent with Vaz's work in pointing out that generalized expectancies are facilitators rather than inhibitors of aggressive behaviour in sport. A similar view has been expressed by the Seville Statement on Violence (1986), which argues that aggression is not genetically programmed but is largely a function of cultural factors. This statement refers to aggression in general and therefore outside Sport, but similar sentiments have been expressed about aggression inside sport [Tenenbaum et al., (1997) ISSP Position Statement on Aggression and Violence].

Problem: Impact of Sex variables on Aggressive Behaviour of All India Inter University sports person. 


\section{Hypothesis}

1. Thereissex difference in aggression of sportsperson.

2. The sportsmen of low SES high aggression and low will to win.

\section{Objectives of Studies}

1. To examine the influences of SES on aggressive behaviour of sportsperson.

2. To verify the significant sex difference in the aggression of sportsperson.

\section{Methodology}

The present investigation pertaining to "Impact of Socioeconomic status on Aggressive Behaviour between male and female Sportsperson" is in the frame of empirical research. The particulars of samples, tools, collection of data and statistical techniques are given below,

\section{Sample}

The total sample consists of 200 sportsmen belonging to high socioeconomic status and low socioeconomic status. The age levels ranging from 19-25 were selected randomly in All India Interuniversity Tournaments held at Thiruchanalpalli the sample design is given below:

Table 1: Sample Design of the study

\begin{tabular}{|c|c|c|c|}
\hline Sex & $\begin{array}{c}\text { High Socioeconomic } \\
\text { Status (HSS) }\end{array}$ & $\begin{array}{c}\text { Low Socioeconomic } \\
\text { Status (LSS) }\end{array}$ & Total \\
\hline Male & 50 & 50 & 100 \\
\hline Female & 50 & 50 & 100 \\
\hline Total & 100 & 100 & 200 \\
\hline
\end{tabular}

\section{Tools}

1. Personal Data Schedule: This is framed to collect information regarding the personal and socio demographic status of the sample.

2. Socio-economic Status Scale: The Socio-economic status scale developed by Bhardwaj and Chavan (1984) has been used in the present study to measure social, educational, professional and economic perspectives of the participants scoring was done according to scoring key given in the manual.

\section{Scoring (Aggressive Behaviour)}

Sports Aggression inventory constructed by a Kumar consists of 25 items in which 13 items are keyed "Yes" and rest of 12 items are keyed "No". For each item score 1 is given to the following responses. Maximum score on the inventory may be 25 on this test and minimum score being 0 .

Statistical Analysis: Keeping the objectives of the study in view, the following statistical techniques were applied. The ttest was used to assess the significant differences between SES and sex with aggressive behaviour.

\section{Results and Discussions}

The major objective of the present study is to assess the influence of socio-psychological correlation on Aggressive Behaviour of sportsmen. Also, the present study intends to measure and probe the impact of sex variables on the other dependent variables like aggressive behaviour. Many distinguished scholars like Rath (1974b) who analysed the relationship between social conditions and personality dimensions, argued that the upper and lower caste groups would show significant differences in their personality make up. Rath also concluded that the personality dimensions are the functional resultants of the socio-economic conditions in which they live.

The hypothesis that there is a significant difference in aggressive behaviour between male and female sportsmen are postulated to test the data. on the rationale that the women were having weak genetically make up, and are having lack of social support, socio-cultural obstacles, orthodox perception and negative attitude towards females, male dominated society and created culture might put her into the secondary and subsidiary role. Under these deprived conditions, she does not show aggression in sport competitions and in her performance.

Table 2: Showing the Mean, SD and 't'" Values of Aggressive Behaviour of the Male and Female Sportsmen

\begin{tabular}{|c|c|c|}
\hline Variables & Male & Female \\
\hline Mean & 11.73 & 11.53 \\
\hline Sd & 2.72 & 3.14 \\
\hline T-value & 0.554 & \\
\hline
\end{tabular}

Table- reveals that mean, SD and ' $t$ ' scores of aggressive behaviours of male and female sportsmen. The mean scores of male and female sportsmen are 11.73 and 11.53 respectively. Th obtained't' value for these two groups is .554 , which is not significant. Therefore, the hypothesis that there is a significant difference in the aggressive behaviour of the male and female sportsmen is not accepted. This is because, irrespective of male and female in the sports field every sportsman should have aggressive behaviour to win the competition. Every competitor develops the sport spirit. The female also has equal exposure to the sport competitions, good training, high will to win, higher level of self-confidence and these factors could have influenced them to adopt the higher level of aggression. Moreover, the fear and frustrations associated with defeat, situational stimulus factors, and nature of the competition might have contributed in stimulating the high aggressive behaviour in female sportsmen on par with the male sportsmen. In conclusion, facilities extended to female are made use properly. Hence, there is no difference in their aggressive and the above-formulated hypothesis is rejected.

\section{Conclusion}

The present result trend reveals that participation of physical education and sports leads emotional stress and cultivates few psychological competencies among sportsperson. But in this study male and female sportsperson of all India interuniversity has exhibited similar intensity of aggressiveness and there is not found any difference in their assertiveness and angriness towards sports situation, it means that sex variables do not influenced on eliciting aggressiveness among the sportsperson.

\section{Reference}

1. Bandura A. Aggression: A social learning analysis. Englewood Cliffs, Prentice-Hall, New Jersey 1973.

2. Bandura A, Ross D, Ross S. Transmission of aggression through imitation of aggressive models. Journal of Abnormal and Social Psychology 1961;63:575-582.

3. Baron RA. Human Aggression, Plenum Publishers, New York 1977.

4. Bateson G. The frustration-aggression hypothesis and culture, Psychological Review 1941.

5. Bennett JC. The irrationality of the catharsis theory of aggression as justification for educators' support of interscholastic football. Perceptual and Motor Skills, 1991;72:415-418.

6. Berkowitz L. Aggression, a social psychological analysis, McGraw Hill, New York 1962. 